\title{
HYPERSPECTRAL IMAGING AS AN ANALYTICAL TOOL FOR THIN SINGLE AND MULTILAYER OXIDES CHARACTERIZATION
}

\author{
Shu Hui Ham ${ }^{1,2,3}$, Morgan Ferté $^{2}$, Cédric Carteret ${ }^{1}$, Gabriel Fricout ${ }^{2}$, Jesus Angulo ${ }^{3}$ \\ ${ }^{1}$ ArcelorMittal Maizières Research (AMMR) \\ ${ }^{2}$ Laboratoire de Chimie Physique et Microbiologie pour l'Environnement (LCPME) Université de Lorraine \\ ${ }^{3}$ Centre de Morphologie Mathématique (CMM) Mines ParisTech
}

\begin{abstract}
Characterization of deposited thin oxide layers using conventional laboratory techniques such as ellipsometry and FTIR requires long sampling time. The hyperspectral technology with a high acquisition rate appears to be an interesting solution for high throughput quantification. To check its capability for this purpose, we develop an analysis protocol in the laboratory using this device to measure thin oxide layers.

In this paper we study the acquisition set-up regarding the incidence angle in reflection mode, the relation of layer thickness and measured signal to enable the quantification and identification of deposited $\mathrm{Al}_{2} \mathrm{O}_{3}$ and $\mathrm{SiO}_{2}$. Analysis results from FTIR, hyperspectral camera and ellipsometry are presented along with calculations based on Fresnel equations. Measured results correspond with the expected theoretical spectra. The study of the detection sensitivity also extends to multilayer oxide.

Overall, the capability of the hyperspectral camera to detect nanometric oxide layers is demonstrated. With this conclusion and in combination with its many advantages, the hyperspectral camera could be used for dynamic analysis of oxide layer.
\end{abstract}

Index Terms-Molecular vibration, hyperspectral technology, thin oxide layer, spectra calculation

\section{INTRODUCTION}

Surface coating by means of plasma technology is a wellestablished technique to modify surface properties. Often, nanometric coatings are deposited to provide specific functions to the bulk material. The characterization of such thin layers requires instruments that are sensitive to the variation of nanoscale thickness.

It is well-known that quantitative and qualitative analysis of the coating such as the thickness measurement and the identification of coating material could be carried out using different commercial solutions existing on the market (spectroscopic ellipsometry, FTIR) but they are mainly local measurements that require long acquisition time and designed for laboratory use. The advent of more advanced hyperspectral technology with a high acquisition rate, compact design and uncooled detectors makes it an interesting solution for high throughput analysis. It is also possible to acquire spectra for each spatial pixel. Owing to that, measurements are not merely restricted to point-wise sampling. Its more compact and robust design compared to most laboratory instruments allows it to be mounted in places lacking space and in conditions less ideal than in a laboratory. These advantages have prompted the investigation of the application of the hyperspectral technology for the purpose of thin oxide layers quantification and identification.

To implement this use case, it is necessary to develop an analysis protocol such as the wavelength range and the optimal set-up. Based on the vibrational spectroscopy of known oxides, $\mathrm{Al}_{2} \mathrm{O}_{3}$ and $\mathrm{SiO}_{2}$, we chose the wavelength range of $8-12 \mu \mathrm{m}$ for this study. From the electromagnetic wave propagation theory, the interaction of a plane wave with a plane boundary could be calculated to give an idea of the relation of layer thickness and absorbance spectrum at various incidence angles. The measured signal from the hyperspectral camera are compared those acquired using FTIR and obtained from calculation for validation.

\section{METHODS AND MATERIALS}

$\mathrm{Al}_{2} \mathrm{O}_{3}$ and $\mathrm{SiO}_{2}$ have been deposited by magnetron reactive sputtering by Institut Jean Lamour, CNRS, Université de Lorraine. In addition to that, $\mathrm{SiOx}$ layers are deposited by chemical vapor deposition (CVD) using a silane precursor. The substrates utilized are standard steel that have been polished prior to deposition. The surface roughness of the polished substrate is measured using an interferometer microscope (Zygo NewView 6200) equipped with an objective lens with $5 x$ magnification. From an analyzed 
surface area of $2.8 \times 2.1 \mathrm{~mm}$, Sa and Sq of the substrates for single layer deposition are inferior to $5 \mathrm{~nm}$ and $8 \mathrm{~nm}$ respectively, while those for bilayer depositions are inferior to $50 \mathrm{~nm}$ and $60 \mathrm{~nm}$ respectively.

The thickness of the deposited layer is obtained by means of spectroscopic ellipsometry employing the HORIBA Jobin Yvon ellipsometer in the spectral range of 245-2100 nm.

Infrared spectra are measured by the Nicolet 8700 Fouriertransform infrared (FTIR) absorption spectroscopy using an angle-variable accessory to vary the incidence angle from $40^{\circ}-60^{\circ}$ in specular reflection mode. The DTGS detector is employed. The pseudo absorbance is calculated from the measured reflectance,

$$
A=-\log _{10}\left(\frac{R_{\text {Sample }}}{R_{\text {reference }}}\right)
$$

where $R_{\text {reference }}$ denotes the reflectance of the uncoated substrate.

A linear hyperspectral camera (Specim Ltd., Oulu, Finland) in the long infrared wavelength range (LWIR), i.e. $8-12 \mu \mathrm{m}$ is employed. This spectral range is chosen due to the presence of the molecular vibration of Si-O and Al-O. The set-up in specular reflection mode is illustrated in Figure 1.

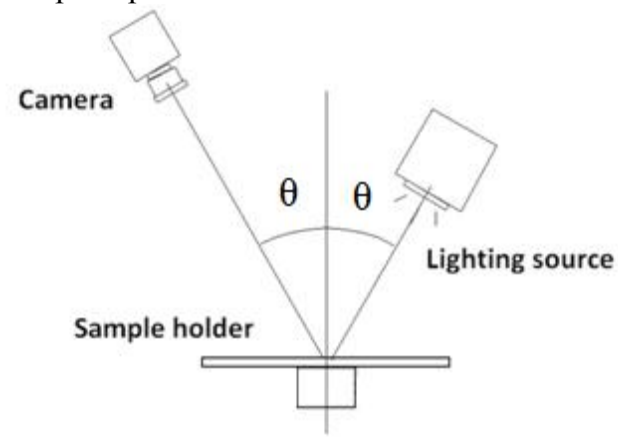

Figure 1: Optical set-up for pushroom acquisition

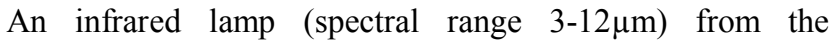
resistance heating of a tubular element composed of nickel/chrome is used as the lighting source.

The removal of background noise is carried out by an acquisition of the dark, $D_{\lambda, x}$ with the lens cap on. Similar to Eq.1, the pseudo absorbance is expressed as:

$A=-\log _{10}\left(\frac{S_{\lambda, x}-D_{\lambda, x}}{R_{\lambda, x}-D_{\lambda, x}}\right)$

$R_{\lambda, x}$ is the signal obtained using a bare substrate while $S_{\lambda, x}$ represents the reflected signal obtained from the deposited samples.

\section{RESULTS}

The area under the curve is known to be proportional to the thickness[1]. On the other hand, it has been demonstrated that the observation angle has an influence on the absorbance intensity due to the traversed thickness[2]. These two factors result in the overall sensitivity of thickness detection. The former relation, if well identified, would give an approximation of layer thickness while the latter is dependent on the acquisition configuration. A study of these two factors allows an optical set-up and quantification of the layer thickness.

For this purpose, the absorbance of a sample with $96 \mathrm{~nm}$ of $\mathrm{SiOx}(\mathrm{CVD})$ has been measured at various incidence angles. After an automatic baseline correction using an algorithm in [3], the absorbance acquired from FTIR and LWIR hyperspectral camera is presented in Figure 2. The LWIR spectrum presented for each configuration is an average of at least 81 spatial pixels.
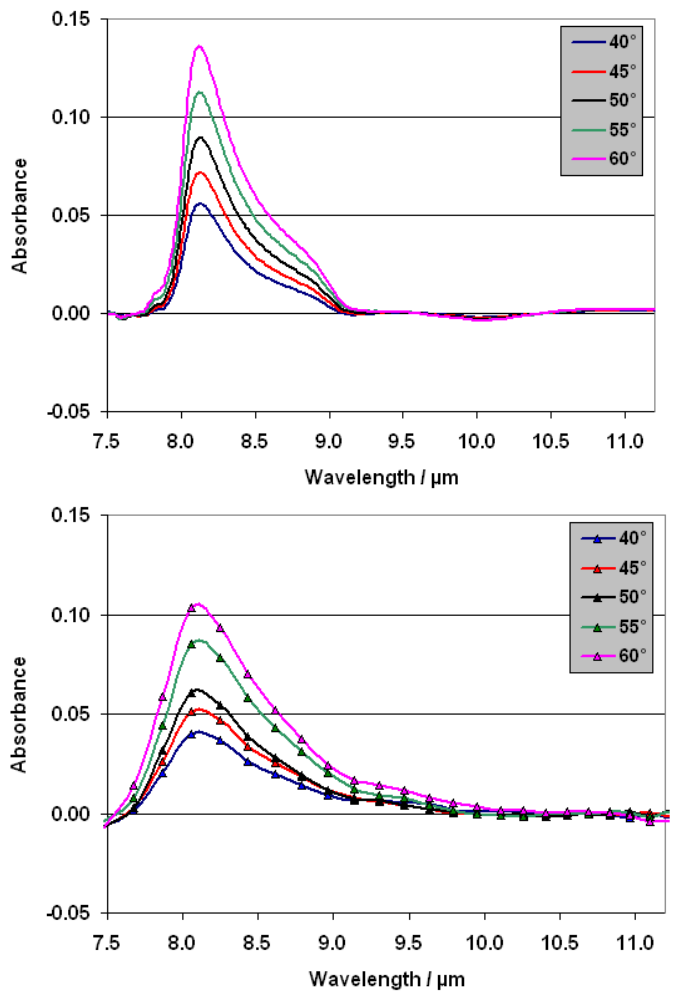

Figure 2: Angular study of 96nm of $\mathrm{SiOx}$ using FTIR (top) and LWIR hyperpsectral camera (bottom)

Absorption at $8 \mu \mathrm{m}$ is attributed to the $\mathrm{Si}-\mathrm{O}-\mathrm{Si}$ asymmetric stretching in longitudinal mode $\left(\mathrm{LO}_{3}\right)$. The area under the 
curve for spectra obtained by both instruments is proportional to the incidence angle due to the increase of distance or thickness traversed with increasing angle of incidence with respect to the surface normal. The slight differences between the spectra obtained from two instruments are explained by the difference in the spectral resolution $(6 \mathrm{~nm}$ and $200 \mathrm{~nm}$ for the FTIR spectrometer and the LWIR camera respectively). The same measurements are also carried out for PVD $\mathrm{SiO}_{2}$ with $97 \mathrm{~nm}$ of thickness. Similar spectra compared to CVD SiOx are obtained.

For the variation of layer thickness at a given incidence angle of $60^{\circ}$, the spectra acquired by the hyperspectral camera for $\mathrm{PVD} \mathrm{Al}_{2} \mathrm{O}_{3}$ and $\mathrm{CVD} \mathrm{SiO}_{\mathrm{x}}$ are shown in Figure 3 and Figure 4. The characteristic absorbance peak for aluminum oxide around $10.8 \mu \mathrm{m}$ is observable.

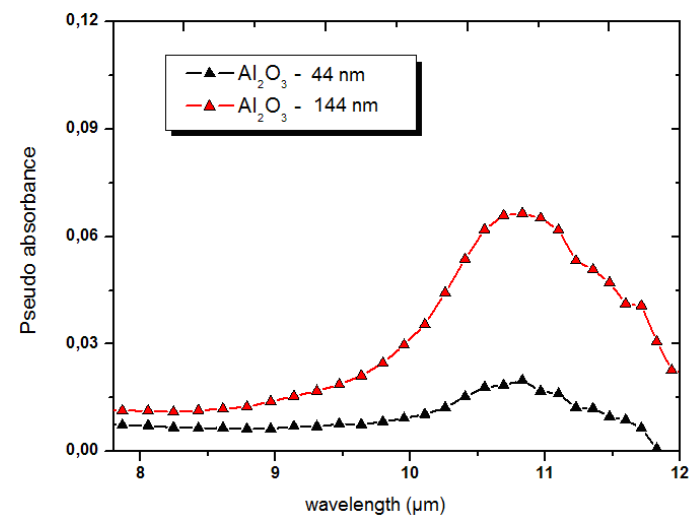

Figure 3: Pseudo absorbance of $\mathrm{Al}_{2} \mathrm{O}_{3}$ layer from hyperspectral camera

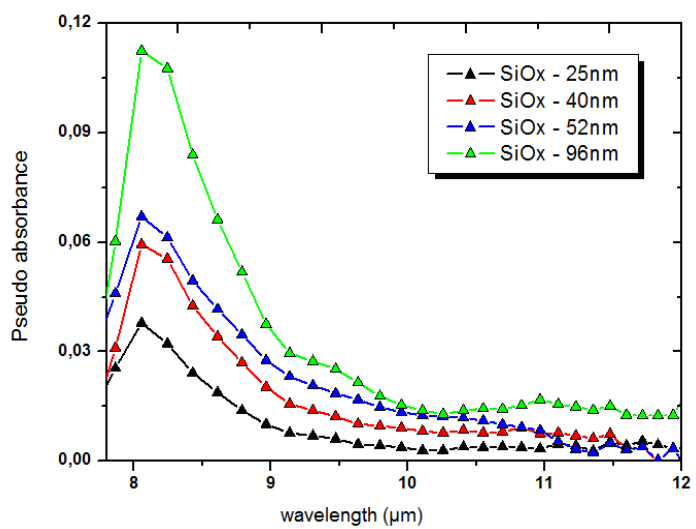

Figure 4: Pseudo absorbance of $\mathrm{SiOx}$ layer from hyperspectral camera

The area under the curve in the vicinity of the peak for $\mathrm{Al}_{2} \mathrm{O}_{3}$ and $\mathrm{SiO}_{2}$ are calculated and plotted against the thickness obtained from ellipsometry and the linear relation is illustrated in Figure 5.
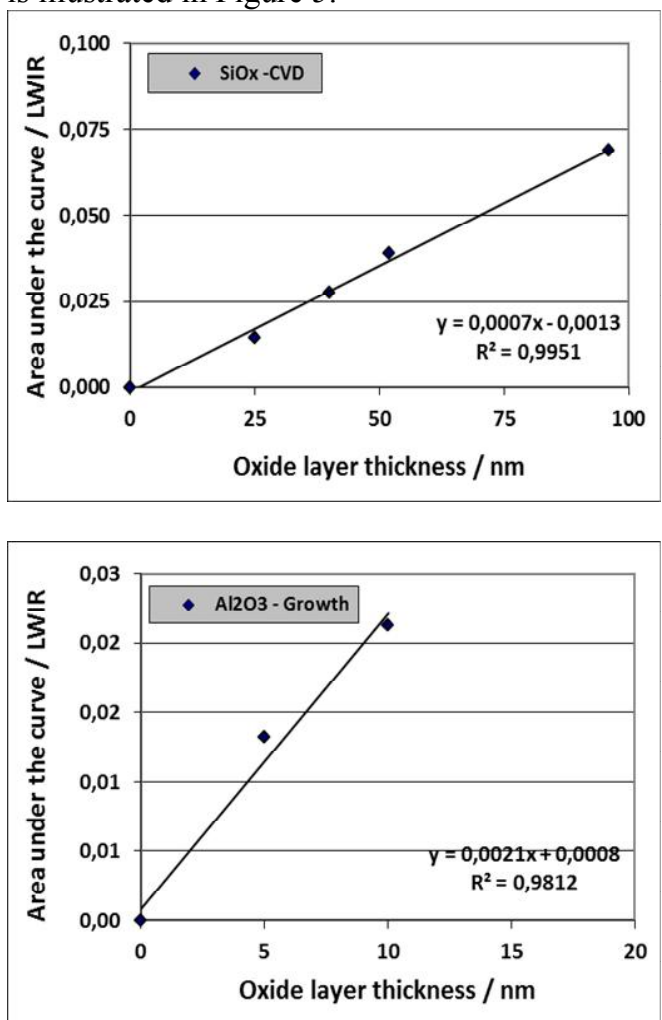

Figure 5: Area under the curve obtained from LWIR hyperspectral camera against thickness for $\mathrm{SiOx}$ (top) and $\mathrm{Al}_{2} \mathrm{O}_{3}$ (bottom)

To validate the analysis results, spectra simulation of the interaction of a plane wave applying the Fresnel equations has been carried out. The sample is modeled using available reference optical constants data[4], [5]. Hence, the substrate for the calculation is replaced by iron. By assuming the incident radiation is randomly polarized, which is in the case of the IR source for the LWIR camera set-up, the reflectivity, $r$ is an average of the two components of polarization $(\mathrm{p}, \mathrm{s})$. Since the oxide layers are deposited on steel substrates, we assume the transmittance is zero and absorptivity, $a=1-r$. The calculated spectra for $100 \mathrm{~nm}$ of $\mathrm{SiO}_{2}$ on $\mathrm{Fe}$ as a function of incidence angle are presented in Figure 6 . From the calculations, the peak position and the general form of the spectra remain similar from $40^{\circ}-60^{\circ}$ incidence angle. Beyond that, the difference in the s- and ppolarized component causes the change of the spectrum shape and peak shift. 


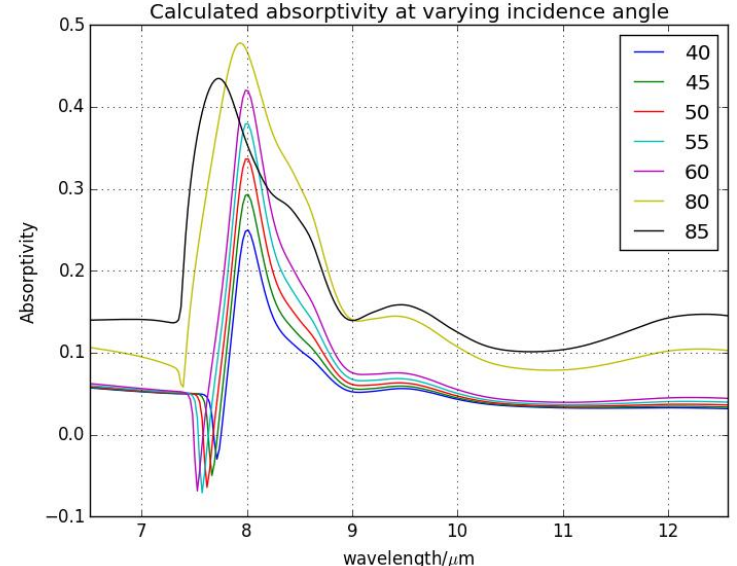

Figure 6: Absorptivity as a function of incidence angle for $100 \mathrm{~nm}$ of $\mathrm{SiO}_{2}$

The absorptivity for bilayer oxides has also been calculated using the matrix method by modeling a system of planeparallel layers [6]. The simulated spectra show no difference as the order of layers is swapped. The absorptivity only depends on the thickness of the constituent oxides. Spectra from FTIR and LWIR hyperspectral camera demonstrate the same trend, i.e. independence of arrangement of bilayers and dependence on thickness. The calculated spectra at $60^{\circ}$ incidence angle based on the thickness input estimated from ellipsometry and the LWIR hyperspectral measurements of the bilayer samples are shown in Figure 7and Figure 8. The theoretical expected spectral shape is obtained by the hyperspectral measurements.

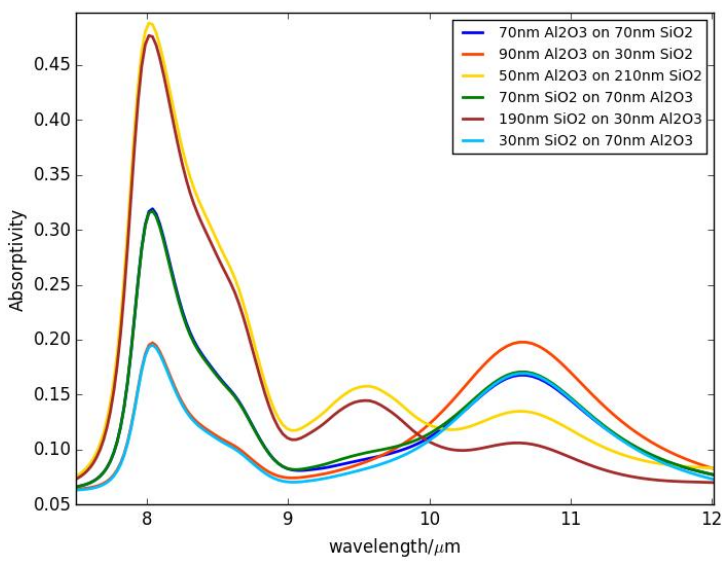

Figure 7: Calculated spectral absorptivity for bilayer oxides at incidence angle $=60^{\circ}$

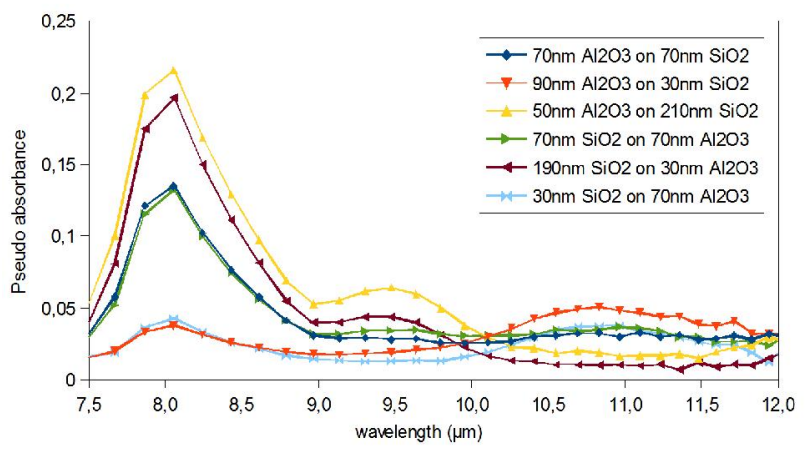

Figure 8: Measurements of bilayer oxide samples from LWIR hyperspectral camera at incidence angle $=60^{\circ}$

\section{CONCLUSION}

Based on measurements and calculations, we obtained a linear relation between the spectral variation (in the range of 7.5 to $9.5 \mu \mathrm{m}$ ) and incidence angle up till $60^{\circ}$ at a given thickness of $\mathrm{SiO}_{2}$. Beyond $60^{\circ}$, spectral form deviates from precedent shape. Thus $60^{\circ}$ incidence angle is employed in the hyperspectral acquisition configuration. Under such setup, the area under the curve is proportional to the thickness variation. The same configuration is carried out for measurements of $\mathrm{Al}_{2} \mathrm{O}_{3}$ layers which also yields linear trend for the spectral quantification around its characteristic absorption peak. Using this knowledge, the thickness of the oxides could be estimated.

In the case of bilayer oxide deposition, the resultant absorbance spectrum is independent of the position of the layer (near surface or near substrate) for given thickness of individual layers. This is shown by both calculations and measurements. The hyperspectral camera is able to detect the spectral variation that is associated with the thickness variation of the individual layers.

From this study, we have established an optimal configuration and identified the relation for the quantification of oxide layer thickness. The results are supported by theoretical calculations. We also show that the hyperspectral camera is sensitive to subsurface oxide species in the case of bilayer measurements. With this knowledge of detectability, the advantages of the hyperspectral technology could be exploited for dynamic thin oxide characterization. 


\section{REFERENCES}

[1] O. Axner, J. Gustafsson, F. M. Schmidt, N. Omenetto, and J. D. Winefordner, 'A discussion about the significance of Absorbance and sample optical thickness in conventional absorption spectrometry and wavelength-modulated laser absorption spectrometry', Spectrochim. Acta Part B At. Spectrosc., vol. 58, no. 11, pp. 1997-2014, Nov. 2003.

[2] B. Harbecke, B. Heinz, and P. Grosse, 'Optical properties of thin films and the Berreman effect', Appl. Phys. A, vol. 38, no. 4, pp. 263-267, Dec. 1985.

[3] C. C. Vincent Mazet, 'Background Removal From Spectra by Designing and Minimising a Non-Quadratic Cost Function', Chemom. Intell. Lab. Syst., vol. 76, no. 2, pp. 121-133, 2005.

[4] J. Kischkat, S. Peters, B. Gruska, M. Semtsiv, M. Chashnikova, M. Klinkmüller, O. Fedosenko, S. Machulik, A. Aleksandrova, G. Monastyrskyi, Y. Flores, and W. T. Masselink, 'Mid-infrared optical properties of thin films of aluminum oxide, titanium dioxide, silicon dioxide, aluminum nitride, and silicon nitride', Appl. Opt., vol. 51, no. 28, pp. 6789-6798, Oct. 2012.

[5] M. A. Ordal, R. J. Bell, R. W. Alexander, L. A. Newquist, and M. R. Querry, 'Optical properties of Al, $\mathrm{Fe}, \mathrm{Ti}, \mathrm{Ta}, \mathrm{W}$, and Mo at submillimeter wavelengths', Appl. Opt., vol. 27, no. 6, pp. 1203-1209, Mar. 1988.

[6] V. P. Tolstoy, I. V. Chernyshova, and V. A. Skryshevsky, Handbook of Infrared Spectroscopy of Ultrathin Films: Tolstoy/Infrared Spectroscopy. Hoboken, NJ, USA: John Wiley \& Sons, Inc., 2003. 